
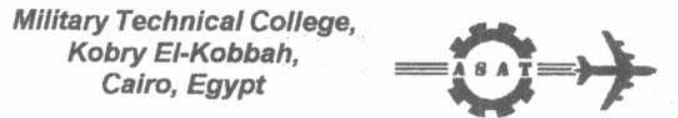

$9^{\text {th }}$ International Conference

On Aerospace Sciences \&

Aviation Technology

\title{
PERFORMANCE OF MODERN GUIDED SYSTEMS IN PRESENCE OF JAMMING SIGNALS
}

Makaryus* A. H., El-Mahdy* A. A., El gamel* S. A.

\section{ABSTRACT}

This paper studies the performance of modern guided systems in presence of a proposed deception jamming technique. The proposed jammer uses the intercepted signal of the guided system to deceive the instantaneous amplitude comparison (IAC) monopulse radar, which is used in the most modern guided system. The performance of the modern guided systems in the presence of jamming for a typical case of radar guided missile is discussed for the following guidance methods: proportional navigation, pure pursuit, and constant bearing. It is found that, the guided system that uses pure pursuit guidance method is the most affected by the proposed jamming technique (largest miss tracking distance). Constant bearing guidance method is the lowest method affected by the proposed jamming technique (lowest miss tracking distance). Proportional navigation, that is the most used guidance method, is moderate affected by the proposed jamming technique. This effect is enough to confuse the guided system and miss track the target.

KEY WORDS: Jamming, and Guided systems

Egyptian Armed Forces 


\section{Introduction}

Most guided system radars are basically specialized tracking radars, located in missiles, whose function is to provide guidance information, which is used to direct the missile to the target. Missile guidance radars can be either active or semi-active types; a combination of both types in a single system is also possible. The trend in guided system guidance appears to be towards active missile seekers. The design principles of active radar missile seekers are identical to those of any tracking radar. However, the size of the antenna aperture is usually small (on the order of five to 15 inches), while the capacity to generate average transmitter power is low [1].

The main task of electronic warfare (EW) against radar guidance systems is to use electromagnetic waves to degrade the guidance performance. This task is performed by generating highly intelligent jamming signals, which are carefully optimized according to the detailed analysis of the guidance system.

This paper is organized as follows. Section II discusses the structure of the proposed jammer. The effect of jamming on the guided system is discussed in section III. Section IV is the simulation of the missile and target trajectories under the three homing guidance methods; these methods are proportional navigation, pure pursuit, and constant bearing with and without jamming. Section $V$ is the conclusion of this paper.

\section{Basic Structure of the Proposed Jammer}

Most modern guided systems use IAC monopulse tracking radar, so the idea of the proposed jamming technique is to introduce an angular error in the target measured angle in this tracking radar.

A proposed self-screen deception jammer is presented. The jammer structure is shown in Fig. 1, it consists of two main parts:

(1) ESM part which intercepts and analyzes the guided system signal, (2) ECM part which produces the required composite jamming signal.

The jamming signal consists of composite $\mathrm{CW}$ pulsed signals with different amplitudes, phases, and delays; the generated jamming signa! has the following form

$$
S_{j}=\sum_{n=1}^{N} A_{n} \cos \left[\omega_{c}\left(t+\tau_{n}\right)+\varphi_{n}\right], \quad 0 \prec t \prec \delta
$$

where $S_{j}$ is the jammer transmitted signal, $A_{n}$ is the amplitude of the nth jamming signal, $\omega_{c}$ is the angular carrier frequency, $\tau_{n}$ is the nth relative time delay (relative to the guided system signal), and $\varphi_{n}$ is the nth relative phase. The jamming signal given in (1) can be written as

$$
S_{f}=\sum_{n=1}^{N} A_{n} \cos \left(\omega_{c} t+\phi_{n}\right), \quad 0 \prec t \prec \delta
$$


where $\phi_{n}=\varphi_{n}+\omega_{c} \tau_{n}$. The expression of $\phi_{n}$ can be written as $\phi_{n}=\omega_{c} \varsigma_{n}$, where $\zeta_{n}$ is another delay. The value of $\zeta_{n}$ must be less than the guided system signal carrier duration $T_{c}$. This condition is necessary to provide multiple false unresolved targets. Each false target corresponds to one of the jamming signals. These false targets lie in the same range gate of the guided system tracking radar. The total effect of this action is that, these targets appear as one target differ from the real target

\section{Effect of Jamming on the Guided System}

The target measured angle in the IAC monopulse radar without jamming is given by the following relation [2],

$$
\frac{1}{\rho} \frac{D}{S}=\theta
$$

where $\theta$ is the target angle (off the antenna bore sight), $D$ is the difference signal, $S$ is the sum signal, and $\rho$ is the proportionality constant. The suffixes $x$ (horizontal channel) and $y$ (vertical channel) for $D$ and $\rho$ are omitted to generalize the previous relation.

The ratio $\mathrm{D} / \mathrm{S}$ is real for a single target and will be complex in the presence of jamming. Now, the effect of the jamming signal given in (1) is discussed on the guided system. Consider the total sum signal (S), which exists in the sum channel that consists of two parts. The first part is $S_{0}$ which is due to the existence of the target at angle $\theta_{o}$ and the second part is $\sum_{n=1}^{N} S_{n}$ which is due to the jamming signal. This jamming signal can be considered as false targets return at angles $\left\{\theta_{1}, \theta_{2}, \ldots \ldots \ldots, \theta_{N}\right\}$. Then the sum signal, in the phasor form, can be written as

$$
S=S_{o}+\sum_{n=1}^{N} S_{n}
$$

similarly the difference channel $(D)$ can be written as

$$
D=D_{o}+\sum_{n=1}^{N} D_{n}
$$

substitute (3) in (5), the difference channel is written as

$$
D=\rho\left[\theta_{o} S_{o}+\sum_{n=1}^{N} \theta_{n} S_{n}\right]
$$


where $\theta_{o}$ is the target angle, $\theta_{1}, \theta_{2}, \ldots \ldots, \theta_{N}$ are the false targets angles [3,4]. At first, consider $n=1$, then if we have a phase difference between the two signals $S_{1}$ and $S_{o}$, then the ratio between the two signals in the phasor form is written as

$$
\frac{S_{1}}{S_{o}}=g e^{j \varphi}
$$

where $g$ is the amplitude ratio between $S_{1}$ and $S_{o}$, and $\varphi$ is the phase difference between them. If there is also a time delay $(\tau)$ between the two signals $S_{1}$ and $S_{o}$, then the ratio $\frac{S_{1}}{S_{o}}$ is written in the phasor form as

$$
\begin{aligned}
\frac{S_{1}}{S_{o}} & =g e^{j \varphi} e^{j \omega_{c} \tau} \\
& =g e^{j \phi}
\end{aligned}
$$

where $\omega_{c}$ is the angular carrier frequency of the signal, and $\phi=\varphi+\omega_{c} \tau$ is the phase difference which will be used all over the work. Similarly for $n=1,2, \ldots, N$, we have

$$
\begin{aligned}
& \frac{S_{1}}{S_{0}}=g_{1} e^{j \phi_{1}} \\
& \frac{S_{2}}{S_{o}}=g_{2} e^{j \phi_{2}} \\
& \frac{S_{N}}{S_{0}}=g_{N} e^{j \phi_{N}}
\end{aligned}
$$

where $g_{n}$ is the nth relative amplitude ratio, and $\phi_{n}$ is the nth relative phase between $S_{n}$ and $S_{o}$. Using (4) and (6), we can write

$$
\frac{1}{\rho} \frac{D}{S}=\frac{\theta_{0} S_{0}+\theta_{1} S_{1}+\ldots \ldots \ldots \ldots+\theta_{N} S_{N}}{S_{o}+S_{1}+\ldots \ldots \ldots \ldots \ldots \ldots \ldots+S_{N}}
$$

by dividing (10) by $S_{o}$, we have 


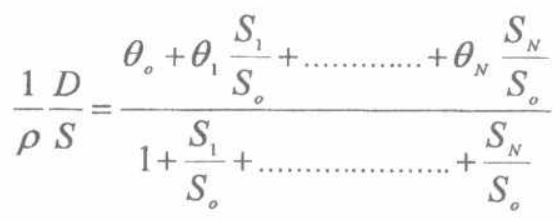

substitute the ratios $\frac{S_{n}}{S_{o}}$, from (9) to (11)

$$
\frac{1}{\rho} \frac{D}{S}=\theta_{j}=\frac{\theta_{o}+\theta_{1} g_{1} e^{j \phi}+\ldots \ldots \ldots \ldots \ldots+\theta_{N} g_{N} e^{j \phi_{N}}}{1+g_{1} e^{j \phi}+\ldots \ldots \ldots \ldots \ldots+g_{N} e^{j \phi_{N}}}
$$

where $\theta_{j}$ is the new complex measured angle due to jamming. This angle results from real target (at angle $\theta_{0}$ ) and $N$ false unresolved targets at angles $\theta_{1}, \theta_{2}, \ldots \ldots, \theta_{N}$.

The effect of jamming on the measured angle $\theta$ can be represented graphically as shown in figure (2). In single target case, the ratio $D / S$ is real since $D$ and $S$ by definition are either inphase or $180^{\circ}$ out of phase. In case of jamming, $D$ and $S$ may have any relative phase and their ratio is therefore complex. Consider a real target and one false target are in the same range cell by definition. Let $S_{o}$ and $S_{1}$ be their individual sum signal phasors, as shown in figure (2). Then the total sum signal is the resultant $S$. Consider the two targets lie on opposite side of the beam axis, the first target being on the side that makes its normalized difference signal positive. Then the difference signal $D_{o}$ is in phase with $S_{o}$, while $D_{1}$ is inphase opposition to $S_{1}$. The total difference $D$ is their resultant. It's clear from the figure that $D$ has a component inphase quadrature with $S$, as well as an inphase component. In other words the ratio $D / S$ is complex [3].

\section{Effect of Jamming on the Missile Target Engagement}

The performance of the jamming technique is studded by simulation, for a typical case of radar guided missile. Three homing guidance methods are used in this paper. These methods are: proportional navigation, pure pursuit, and constant bearing. We consider vertical plane motion and apply the kinematics equations together with the law of guidance equations. The following initial conditions are used, the initial line of sight length $\left(D_{o}\right)=10 \mathrm{Km}$, the initial line of sight angle $\left(\varepsilon_{o}\right)=20^{\circ}$, the missile velocity $\left(v_{M}\right)=800 \mathrm{Km} / \mathrm{hour}$, the target velocity $\left(v_{T}\right)=600 \mathrm{Km} / \mathrm{hour}$, and 
the angle of the target velocity $\left(\theta_{T}\right)=20^{\circ}$. The flow chart of the simulation model of the target and missile flight trajectories is shown in Fig.3. In Fig.4, we plot the target and missile trajectories for the previous mentioned guidance methods. It's clear that the length and curvetion of the missile trajectory depends on the guidance method as well as the time of flight to the hit point.

The idea of introducing the jamming in the missile target engagement as follows, we calculate next the LOS angle under jamming $\varepsilon_{j_{(1+1)}}$ from the following; first we calculate the angle $\varepsilon_{i+1}^{\prime}$, between the new position of the real target $\left(X_{T_{(i+1)}}, Y_{\tau_{(i+1)}}\right)$ and the position of the jammed missile $\left(X_{M_{f_{h}}}, Y_{M_{h}}\right)$

$$
\varepsilon_{i+1}^{\prime}=\tan ^{-1}\left(\frac{Y_{T_{i+1}}-Y_{M_{11}}}{X_{T_{i+1}}-X_{M_{n}}}\right)
$$

The difference angle $e \varepsilon_{i+1}$, between the angle $\varepsilon_{i+1}^{\prime}$ and the last LOS jammed angle $\varepsilon_{j_{1}}$ is given by

$$
e \varepsilon_{i+1}=\varepsilon_{i+1}^{\prime}-\varepsilon_{j_{t}}
$$

This difference angle $e \varepsilon_{i+1}$ represents the error of the false target off the center of the beam, then this angle is introduce in the jamming equation to evaluate the jammed error angle $e \varepsilon_{j_{(i+1)}}$. Then the new jammed LOS angle $\varepsilon_{j_{(i+1)}}$ is calculated by adding the previous LOS angle $\varepsilon_{j_{t}}$ to the jammed error angle $e \varepsilon_{j_{(+1+1}}$

$$
\varepsilon_{j_{(1+1)}}=\varepsilon_{j_{i}}+e \varepsilon_{j_{(i+1)}}
$$

This processing repeated along the target and missile flights till the difference between the angles $\varepsilon, \varepsilon$, becomes greater than the antenna half-power beam width. At this instant the missile seeker loses the target, which is out of its field of view. The target senses the instant its outside the radar beam of the seeker. The pilot can start maneuvering away from the missile. Usually the missile guidance and control systems include a memory device, such that if the seeker loses tracking the target, it will keep the final value of its velocity vector angle. If the fuze dose not sense the target for certain time, the missile will be self destroyed. This situation is simulated using final data of missile flight. We suppose that the pilot will keep the aeroplane angular velocity $\omega_{\text {escape }}=0.05 \mathrm{rad} / \mathrm{sec}$., the previous situation the missile and target engagement is shown in Figs. 5,7, and 9 for the three guidance methods. In Figs. 6,8 , and 10 the missile and target distance is plotted allover the missile flight (considered equal $60 \mathrm{sec}$.) for the cases of guidance methods. 


\section{v. Conclusion}

A jamming technique is proposed to repeat the guided system signal with change in its amplitude and phase (deception jamming signal) to produce an angular error in the guided system seeker. This error changes for different values of amplitude ratio and phase difference w.r.t the guided system signal. The performance of the jamming technique is measured for a typical case of active radar guided missile. It is found that, the missile that uses pure pursuit guidance method is the most affected by the proposed jamming technique (largest miss tracking distance). Constant bearing guidance method is the lowest method affected by the jamming technique (lowest miss tracking distance). Proportional navigation, that is the "most used guidance method, is moderate affected by the jamming technique. This effect is enough to confuse the missile and miss track the target.

\section{REFRENCES}

[1] George W. Ewell and Neal T. Alexander, Principle of Modern Radar, Artech house, Inc. 1997.

[2] Peyton Z. peebles, JR., Radar Principles, John Wiley and Sons, Inc., 1998.

[3] Sammuel M. Sherman "Complex Indicated Angles Applied to Unresolved Radar Target and Multipath ", IEEE Trans. on Aerospace and Electronic Systems,
vol. AES-7, No. 1, January 1971.

[4] Peyton Z. Peebles, JR. and L. Goldman, JR. " Radar Performance with Multipath Using the Complex Angle ", IEEE Trans. on Aerospace and Electronic Systems, vol. AES-7, No. 1. January 1971. 


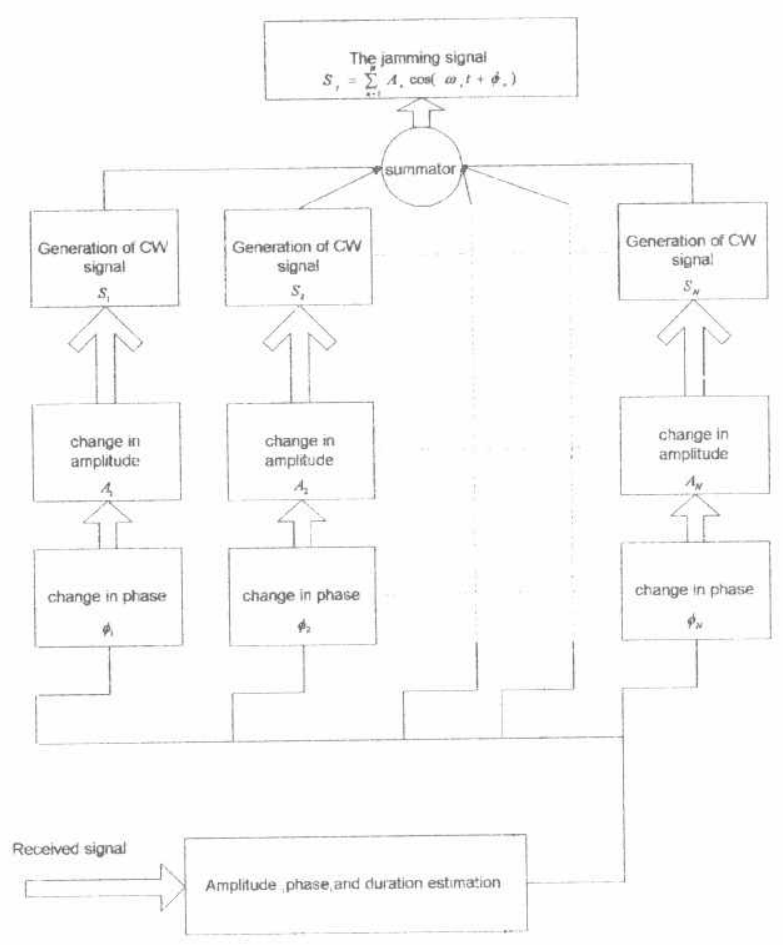

Fig. 1. The structure of the proposed jarnmer

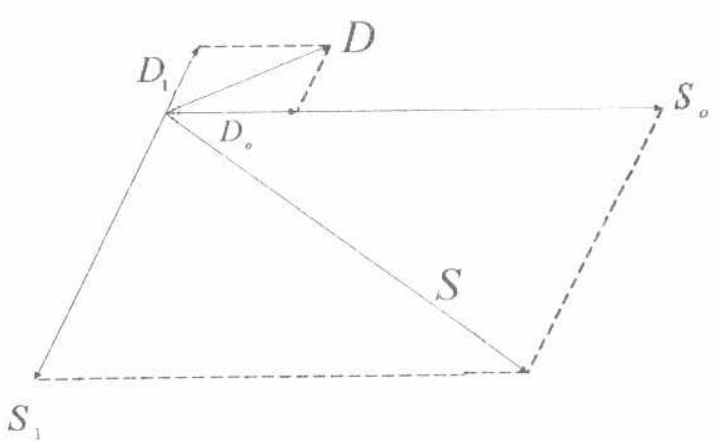

Fig.2. Phasor representation of the resultant sum and difference signal 


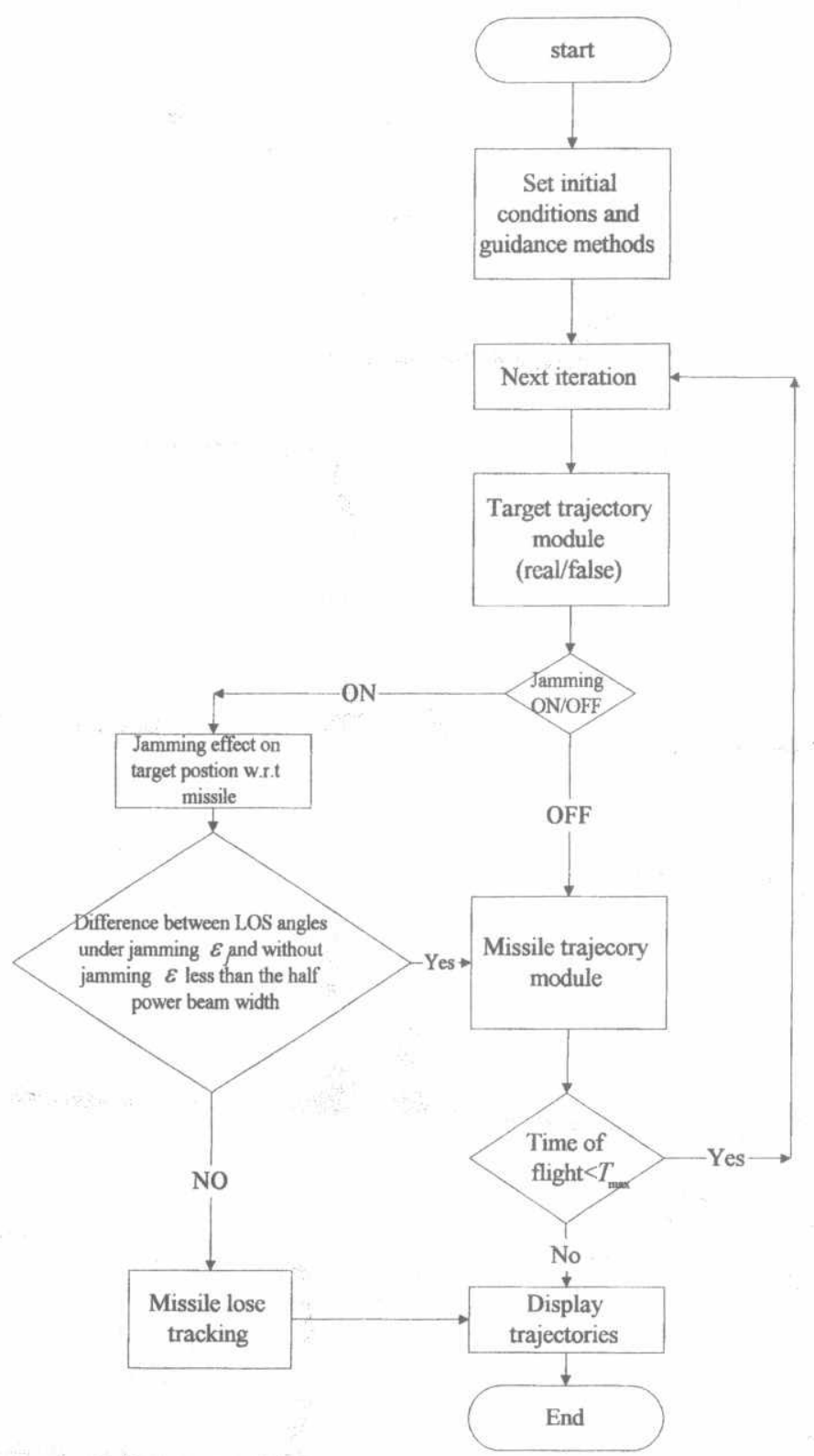

Fig.3. Flow chart of the simulation model 


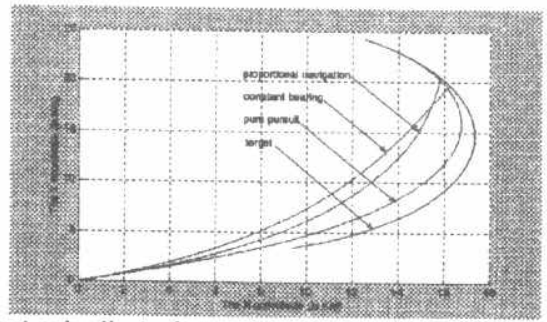

Fig.4. The target-missile trajectories under different guidance methods

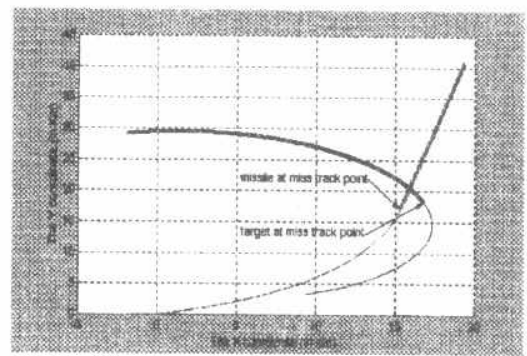

Fig.5. The target-missile trajectories under jamming for proportional navigation guidance method after point of miss track

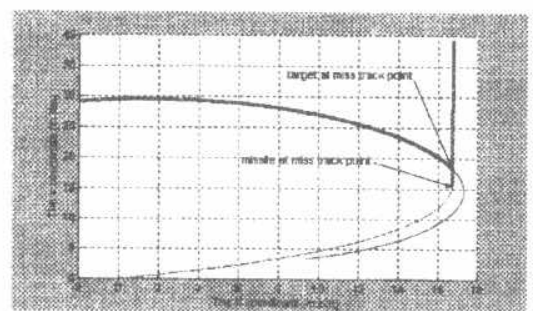

Fig.7. The target-missile trajectories under jamming for pure pursuit guidance method after point of miss track

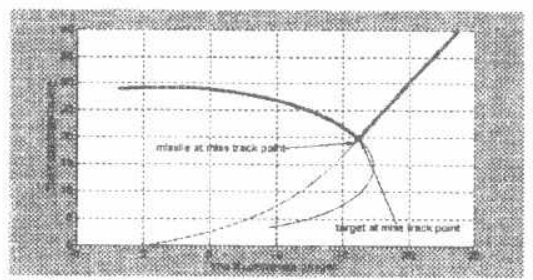

Fig.9. The target-missile trajectories under jamming for constant bearing guidance method after point of miss track

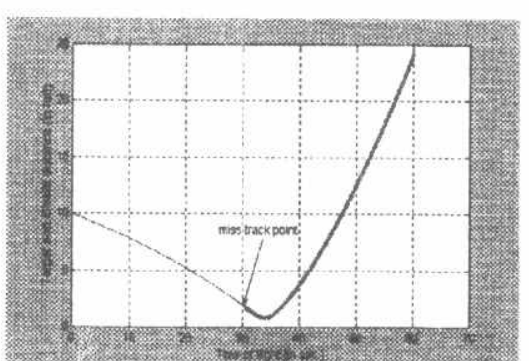

Fig.6. Target and missile distance during the missile flight

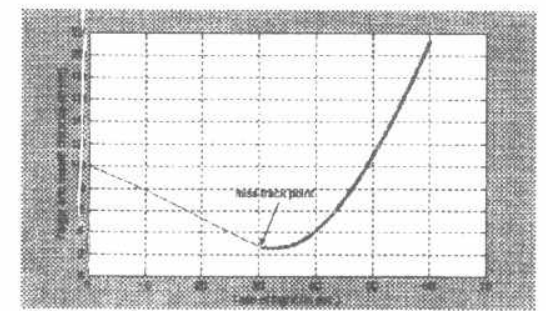

Fig.8. Target and missile distance during the missile flight

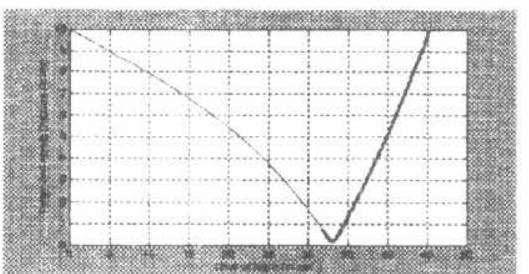

Fig. 10. Target and missile distance during the missile flight 